\title{
Salivary Cortisol: A Biomarker for Stress Indicator in Children
}

\author{
Prathibha Rani $\mathrm{S}^{1}$, Amrutha $\mathrm{C}^{2}$, Anantharaj $\mathrm{A}^{3}$, Praveen $\mathrm{P}^{4}$, Sudhir $\mathrm{R}^{5}$
}

\begin{abstract}
Aim and objective: To assess the child's anxiety level for various dental procedures using a standard questionnaire and by measuring the salivary cortisol level.

Study design: Cross-sectional study.

Materials and methods: A sample of 24 healthy children (8-10 years) visiting the Department of Pedodontics and Preventive Dentistry, DAPM RV Dental College, Bengaluru were selected. They were divided into three groups: group I-eight children having their first dental visit, group II-eight children requiring oral prophylaxis after their first visit, group III-eight children requiring extraction of 1 or 2 teeth after their first visit. Levels of dental anxiety were assessed in children using the State-Trait Anxiety Inventory for Children State (STAIC-S) before and after the treatment. Salivary cortisol was assessed in children before and after the treatment using the ELISA test. Statistical analysis used: Student paired $t$-test was used to compare the mean anxiety score and cortisol levels between pre- and post-time intervals for different procedures within the child group.
\end{abstract}

Results: In children, the mean anxiety score was reduced after the treatment. There was a proportionate decrease in the mean anxiety level among children irrespective of the procedure. Also, there was a reduction in the cortisol level from pre- to post-procedure among children.

Conclusion: Assessment of cortisol level in children could be a significant factor that can be used as one of the physiological parameters for various dental procedures.

Keywords: Anxiety scales, Children, Dental anxiety, Oral cavity, Salivary cortisol, State-trait anxiety score.

CODS Journal of Dentistry (2020): 10.5005/jp-journals-10063-0064

\section{INTRODUCTION}

Dental treatment can be a major source of anxiety and fear among patients and is considered to be one of the most stressful environments for many people, especially children. ${ }^{1}$ Dental fear and anxiety act as barriers for the effective and efficient management of the child for the dental team.

Measuring physiological distress can give dentists valuable information about a child's anxiety and program directed toward behavior modification. ${ }^{2}$

Cortisol is a hormonal marker of stress which has received considerable attention in studies of psychological and physical health. ${ }^{3}$ Fear and anxiety increase the activity of the hypothalamicpituitary axis (HPA) which in turn enhances the secretion of cortisol. A major methodological breakthrough for psychological research was achieved when it was shown that salivary cortisol levels reliably reflected circulating hormone levels which enabled assessment of adrenal activity across the day in a non-invasive manner. ${ }^{3}$

Self-reported questionnaires are frequently used to assess children's anxiety and dental fear. The state-trait anxiety inventory for children's state form [State-Trait Anxiety Inventory for ChildrenState (STAIC-S)] was developed by Spielberger and contains 20 items, and it is one of the most frequently used self-report instruments for evaluating children's anxiety. The STAIC-S offers high reliability and satisfactory validity. ${ }^{4}$

Although studies have assessed the internal physiological changes during dental treatment in pediatric dental patients, there is a paucity of data on the physiological distress experienced by children during dental treatment. Thus, in the present study, the subjective assessment of anxiety in children during various dental procedures was done using STAI-S questionnaire and objective assessment by measuring the salivary cortisol level.

The objectives of this study are to assess and correlate the child's anxiety level during various dental procedures using
${ }^{1-5}$ Department of Pediatric and Preventive Dentistry, DAPM RV Dental College and Hospital, Bengaluru, Karnataka, India

Corresponding Author: Prathibha Rani S, Department of Pediatric and Preventive Dentistry, DAPM RV Dental College and Hospital, Bengaluru, Karnataka, India, Phone: +91 9886391166, e-mail: prathibha.rvdc@rvei.edu.in

How to cite this article: Rani SP, C Amrutha, A Anantharaj, et al. Salivary Cortisol: A Biomarker for Stress Indicator in Children. CODS J Dent 2020;12(1):7-10.

Source of support: Nil

Conflict of interest: None

a questionnaire and salivary cortisol level and investigate the association between these variables.

\section{Materials and Methods}

\section{Materials}

- Questionnaire.

- Eppendorf tubes.

- Human cortisol ELISA kit (ab154996)-Abcam.

\section{Sources of Data}

Twenty-four healthy children (8-10 years) visiting the Department of Pedodontics and Preventive Dentistry, DAPM RV Dental College, Bengaluru were selected.

\section{Inclusion Criteria}

- Children aged 8-10 years.

- Children with the first dental visit.

- Children requiring oral prophylaxis.

- Children requiring extraction of 1 or 2 teeth. 


\section{Exclusion Criteria}

Children:

- Having any history of systemic diseases.

- On corticosteroid therapy.

- Who are medically compromised.

- With severe sensory-motor impairment.

- With abnormal psychological development.

The purpose of the study was explained to the children and their parents who signed a written consent to participate. This study was approved by the Ethical review board.

\section{Groups}

Group I-Eight children having their first dental visit.

Group II-Eight children requiring oral prophylaxis after their first visit.

Group III-Eight children requiring extraction of 1 or 2 teeth after their first visit.

\section{Methodology}

\section{Self-reported Questionnaire}

Levels of dental anxiety were assessed in children using the STAIC-S before and after treatment. In this study, we used only the state anxiety scale to compare anxiety levels between pre- and posttreatment in children. The STAIC-S scale consists of 20 statements that ask children how they feel at a particular moment. Children respond to STAIC-S on a three-point rating scale (1-3) with three alternative choices. Scores on the STAIC-S subscale range from 20 to 60 with a higher score indicating greater anxiety. The STAI-S provides measures of anxiety for adolescents and adults and consists of 20 statements that evaluate how the respondent feels at the present moment. Each STAI-S item is rated on a four-point scale (1-4) with four alternative choices. Scores on the STAI-S subscale range from 20 to 80 , with a higher score indicating greater anxiety. ${ }^{2}$

\section{Measurement of Salivary Cortisol Level}

Salivary samples were collected 10 minutes before and 30 minutes after each dental treatment. All procedures were scheduled for morning appointments, between 9 am to $11 \mathrm{am}$. Unstimulated saliva about 1-2 $\mathrm{mL}$ was collected from child and mother after rinsing mouth by half glass of water, and expectorating into a disposable plastic container. Disposable plastic containers containing saliva were tightly closed, labeled, and stored in the refrigerator at $4^{\circ} \mathrm{C}$ and transported to the laboratory. Salivary cortisol level was assessed by immunoassay kit.

\section{Procedure}

For children, the levels of dental anxiety and pretreatment salivary cortisol will be recorded in a separately allocated room, whereas post-treatment state anxiety and salivary cortisol will be measured in the dental treatment room.

\section{Results}

There was no difference in the mean anxiety scores before and after the completion of different procedures (Table 1 and Fig. 1).

There was no statistically significant difference in the cortisol level before and after the first visit and the oral prophylaxis procedure among children. However, a significant difference was seen in the mean cortisol level after the extraction procedure in children. The mean cortisol level among children increased significantly soon after the extraction (Table 2 and Fig. 2).

Intragroup comparison results showed that there was a significant difference in the anxiety score pre- and post-procedures among the children. There was a significant reduction in the anxiety scores after the examination, oral prophylaxis, and extraction.

Intragroup comparison of mean cortisol level for the first visit did not show a significant difference among children. However, there was a statistically significant difference after prophylaxis and extraction. The mean cortisol level decreased significantly after oral prophylaxis but increased to a significant level after the extraction (Fig. 2).

\section{Discussion}

Dental treatment is often considered anxiety-producing and stressful. ${ }^{5}$ The anxiety level can be different for different dental procedures. Non-invasive procedures like examination and oral prophylaxis may produce lesser anxiety when compared to invasive procedures like pulp therapy and extractions. In our study, procedures representative of the non-invasive and invasive methods were chosen to facilitate the feasibility of sample selection.

A child's behavior can make it challenging to provide effective dental treatment. In this study, anxiety was investigated as a subjective assessment and physiologic distress as an objective assessment in children before and after pediatric dental procedures.

State-Trait Anxiety Inventory for Children was initially developed as a research tool for the study of anxiety in elementary school children. While especially constructed to measure anxiety in 9- to 12-year-old children, STAIC may also be used with younger children with average or above reading ability and with older children who are below average in ability. It is comprised of separate, self-report scales for measuring two distinct anxiety concepts-state anxiety (A-State) and trait anxiety (A-Trait). ${ }^{6}$ In our study, we assessed the state anxiety because it is designed to measure the transitory anxiety states that are subjective, consciously perceived feelings of apprehension, tension, and worry that vary in intensity and fluctuate over time.

Table 1: Comparison of mean anxiety scores in a child between pre- and post-Rx for a different procedures using Student paired $t$-test

\begin{tabular}{|c|c|c|c|c|c|c|c|c|c|}
\hline Subjects & Procedure & Time & $N$ & Mean & $S D$ & SEM & Mean Diff & $t$ & pvalue \\
\hline \multirow[t]{6}{*}{ Child } & First visit & Pre & 8 & 35.0 & 5.7 & 2.0 & 7.8 & 3.779 & $0.007^{*}$ \\
\hline & & Post & 8 & 27.3 & 2.3 & 0.8 & & & \\
\hline & $\begin{array}{l}\text { Oral } \\
\text { prophylaxis }\end{array}$ & Pre & 8 & 37.6 & 5.4 & 1.9 & 6.0 & 6.350 & $<0.001^{*}$ \\
\hline & & Post & 8 & 31.6 & 4.4 & 1.6 & & & \\
\hline & Extraction & Pre & 8 & 47.4 & 7.4 & 2.6 & 20.9 & 8.201 & $<0.001^{*}$ \\
\hline & & Post & 8 & 26.5 & 2.6 & 0.9 & & & \\
\hline
\end{tabular}

*Denotes that the values are statistically significant 


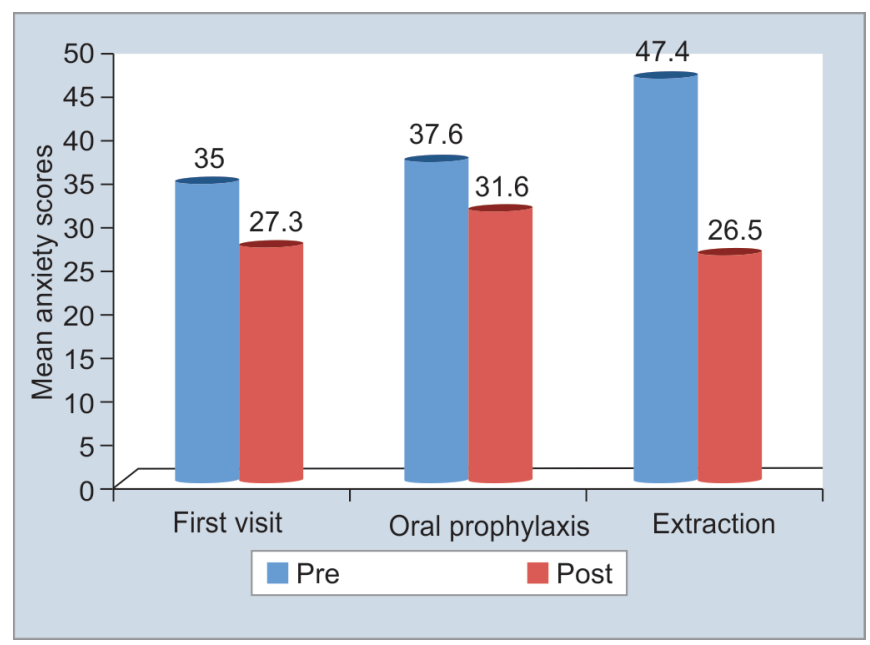

Fig. 1: Comparison of mean anxiety scores in children between pre- and post-Rx for a different procedures

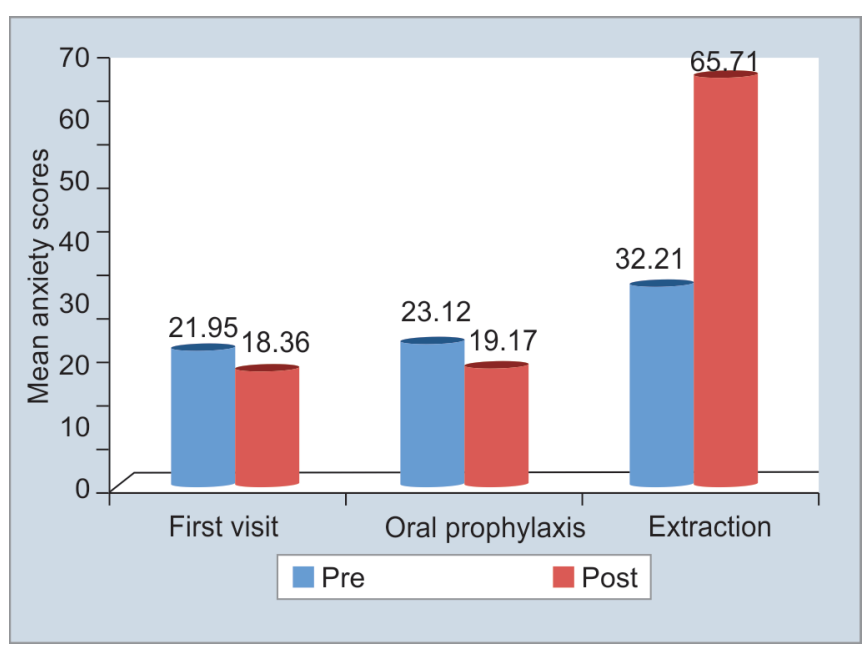

Fig. 2: Comparison of mean cortisol levels in children between pre- and post-Rx for a different procedures

Table 2: Comparison of mean cortisol levels in a child between pre- and post-Rx for a different procedures using Student paired $t$-test

\begin{tabular}{|c|c|c|c|c|c|c|c|c|c|}
\hline Subjects & Procedure & Time & $N$ & Mean & $S D$ & SEM & Mean Diff & $t$ & $p$ value \\
\hline \multirow[t]{6}{*}{ Child } & First visit & Pre & 8 & 21.95 & 5.0 & 1.8 & 3.59 & 1.988 & 0.09 \\
\hline & & Post & 8 & 18.36 & 5.4 & 1.9 & & & \\
\hline & $\begin{array}{l}\text { Oral } \\
\text { prophylaxis }\end{array}$ & Pre & 8 & 23.12 & 5.2 & 1.8 & 3.95 & 2.572 & $0.04^{*}$ \\
\hline & & Post & 8 & 19.17 & 4.1 & 1.5 & & & \\
\hline & Extraction & Pre & 8 & 32.21 & 6.5 & 2.3 & -33.50 & -3.915 & $0.006^{*}$ \\
\hline & & Post & 8 & 65.71 & 22.7 & 8.0 & & & \\
\hline
\end{tabular}

*Denotes that the values are statistically significant

Assessment of free cortisol in saliva has become an increasingly important tool in stress research because it has several advantages over the measurement of cortisol in blood or urine. Of prime interest is the non-invasiveness of sampling and the ability to obtain samples at short intervals from subjects. The measurement of cortisol is the method of choice for field studies or ambulatory assessment because medical personnel or laboratory facilities are not required for collecting and storing the samples. ${ }^{7}$ All procedures were scheduled for morning appointments, between 9 am and 11 am to avoid the diurnal variations.

In children, the increased anxiety score and cortisol level eventually decreased after the completion of non-invasive procedures like screening and prophylaxis. This was in accordance with the findings of Miller et al. who investigated the adrenal response to various dental treatments in healthy adults and reported that cortisol levels measured at the start of dental procedure decreased in patients undergoing non-invasive dental treatments. ${ }^{3,8}$

In the extraction group, the mean anxiety score, as well as salivary cortisol level in the preoperative stage, was high compared to other groups, this may be because of fear of local anesthesia and surgical procedure. But the cortisol level in children increased after extraction even though the anxiety level has come down: this may be because the stress associated with extraction persisted to a postoperative period which may be suggestive of loss of a tooth generated fear. ${ }^{5}$
Measuring a child's anxiety and physiological distress can give pedodontists valuable information to provide tailored behavior modification methods. However, the limitation of the study was limited sample size, procedural variations were not completely assessed.

\section{Recommendations}

Comparison of maternal anxiety with child's anxiety should be measured.

Larger sample should be considered.

Behavior rating of the child was not assessed.

\section{Conclusion}

Within the limitation of the study, we would like to conclude that:

There was a proportionate decrease in the anxiety and cortisol levels in children after procedures like first visit and oral prophylaxis. However, there was a statistically significant increase in cortisol level in children after the extraction procedure indicating persistent anxiety level related to loss of a tooth.

\section{References}

1. Ter Horst G, De Wit CA. Review of behavioural research in dentistry 1987-1992: dental anxiety, dentist-patient relationship, 
compliance and dental attendance. Int Dent J 1993;43(3 Suppl 1): 265-278.

2. Karibe H, Aoyagi-Naka K, Koda A. Maternal anxiety and child fear during dental procedures: a preliminary study. J Dent Child (Chic) 2014;81(2):72-77.

3. Seltzer MM, Greenberg JS, Hong J, et al. Maternal cortisol levels and behavior problems in adolescents and adults with ASD. J Autism Dev Disord 2010;40(4):457-469. DOI: 10.1007/s10803-0090887-0.

4. Nilsson S, Buchholz M, Thunberg G. Assessing children's anxiety using the modified short state-trait anxiety inventory and talking mats: a pilot study. Nurs Res Pract 2012;2012:932570. DOI: 10.1155/2012/932570.
5. Patil SJ, Shah PP, Patil JA, et al. Assessment of the changes in the stress-related salivary cortisol levels to the various dental procedures in children. J Indian Soc Pedod Prev Dent 2015;33(2):94-99. DOI: 10.4103/0970-4388.155116.

6. Papay JP, Costello RJ, Hedl JJ, et al. Effects of trait and state anxiety on the performance of elementary school children in traditional and individualized multiage classrooms. J Educ Psychol 1975;67(6):840846. DOI: 10.1037/0022-0663.67.6.840.

7. Kirschbaum C, Hellhammer DH. Salivary cortisol. In: Fink G, ed. Encyclopedia of stress, vol. 3, San Diego, CA: Academic Press; 2000. pp. 379-384.

8. Miller CS, Dembo JB, Falace DA, et al. Salivary cortisol response to dental treatment of varying stress. Oral Surg, Oral Med, Oral Pathol, Oral Radiol Endodontol 1995;79(4):436-441. 